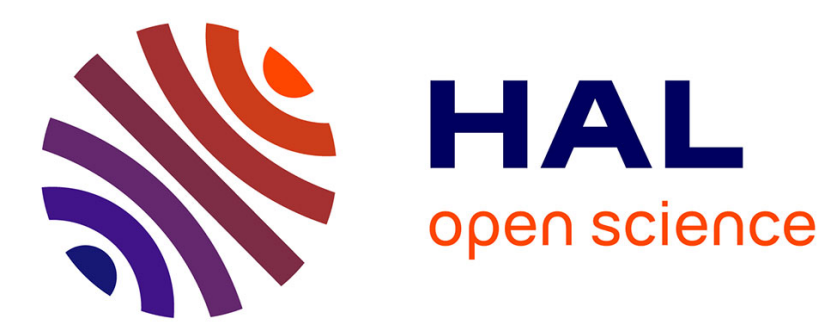

\title{
CDEEC: A Connectivity Degree-Based Energy Efficient Clustering Protocol for Wireless Sensor Networks
}

Sofiane Moad, Mohammad Abdul Azim, Nizar Bouabdallah, Rami Langar

\section{To cite this version:}

Sofiane Moad, Mohammad Abdul Azim, Nizar Bouabdallah, Rami Langar. CDEEC: A Connectivity Degree-Based Energy Efficient Clustering Protocol for Wireless Sensor Networks. IFIP Wireless Days, Oct 2011, Niagara Falls, Canada. 10.1109/WD.2011.6098172 . hal-00715702

\section{HAL Id: hal-00715702 https://hal.inria.fr/hal-00715702}

Submitted on 9 Jul 2012

HAL is a multi-disciplinary open access archive for the deposit and dissemination of scientific research documents, whether they are published or not. The documents may come from teaching and research institutions in France or abroad, or from public or private research centers.
L'archive ouverte pluridisciplinaire HAL, est destinée au dépôt et à la diffusion de documents scientifiques de niveau recherche, publiés ou non, émanant des établissements d'enseignement et de recherche français ou étrangers, des laboratoires publics ou privés. 


\section{CDEEC: A Connectivity Degree-Based Energy Efficient Clustering Protocol for Wireless Sensor Networks}

\author{
Sofiane Moad \\ IRISA/INRIA \\ Campus de Beaulieu \\ 35042 Rennes Cedex France \\ Email: Sofiane.Moad@irisa.fr \\ Rami Langar \\ LIP6 Computer Science Laboratory \\ 4 place Jussieu 75005 Paris, France \\ Email: rami.langar@lip6.fr
}

University of Pierre et Marie Curie-UPMC,

\author{
Mohammad Abdul Azim \\ IRISA/INRIA \\ Campus de Beaulieu \\ 35042 Rennes Cedex France \\ mazim_2002@yahoo.com.au
}

\author{
Nizar Bouabdallah \\ IRISA/INRIA \\ Campus de Beaulieu \\ 35042 Rennes Cedex France \\ Email: Nizar.Bouabdallah@inria.fr
}

\begin{abstract}
In this paper we propose CDEEC, a Connectivity Degree-based and Energy Efficient Clustering protocol, for wireless sensor networks in order to achieve further energy conservation while extending the network lifetime. The main idea behind our proposal is to consider, in addition to the information about the nodes' residual energy, the specific topology characteristics of the network, such as the connectivity degree, in the selection process of the cluster heads. To evaluate the performance of our proposal, we compare it to classical HEED-based (Hybrid, Energy-Efficient Distributed Clustering) networks. Simulation results show that significant energy conservation can be achieved.
\end{abstract}

keywords: Wireless sensor networks, energy efficiency, clustering.

\section{INTRODUCTION}

Wireless sensor networks (WSNs) consist of tiny nodes capable of sensing, computing and communicating [1]. The main role of WSNs is to collect and route data back to a collection point known as the Base Station (BS). Due to large network sizes, limited power supplies and inaccessible remote deployment environments, WSNs introduce new challenges compared to traditional wireless networks [2]. One of the major concerns is the short lifespan of such networks. To extend the network lifetime, designing energy-efficient protocols is critical. Exploiting clustering is one of the most interesting ways in achieving such an objective [3]. A number of clusterbased protocols have been developed to make these networks practical and efficient [4].

Cluster-based protocols were originally proposed concerning wireline networks in order to alleviate scalability issues. However, clustering is used in WSNs for efficient energy consumption in order to extend network lifetime. Typically, nodes with relatively higher residual energy can operate as Cluster Heads (CHs). They send aggregated information from

978-1-4577-2028-4/11/\$26.00 C 2011 IEEE the nodes with lower residual energy, which are only used to perform sensing in the proximity of the target. This helps to balance energy consumption inside the network, and thus improves the overall network lifetime. To achieve energy efficiency, a multi-hop routing protocol can be integrated into the cluster-based protocols in two ways. The first way concerns integrating an intra-communication protocol within a cluster [5]. Instead of sending data directly to the CHs, a routing protocol is incorporated within a cluster to achieve such an objective. The second way concerns integrating an inter-communication protocol between clusters [7]. In this case, $\mathrm{CHs}$ organize themselves into a routing architecture to deliver data to the BS, instead of direct data transmission to the BS.

To this regard, various clustering protocols have been proposed in the literature [3]- [4]. In order to reduce the signalling overhead, the proposed protocols usually use heuristic probabilistic techniques, in which a node becomes a $\mathrm{CH}$ with a certain probability, as opposed to deterministic techniques, which require sending overhead messages for the $\mathrm{CH}$ 's election.

In this paper, we chose the HEED protocol [8], one of the well-known probabilistic clustering protocol in WSNs, to extend the network lifetime. In HEED, the probability of becoming a $\mathrm{CH}$ is based on the nodes' current residual energy. However, in HEED no emphasis is made on the specific network topology characteristics, such as the degree of connectivity in the selection of CHs. The main idea behind our proposed CDEEC protocol is then to consider, in addition to the information about the nodes' residual energy, the degree of connectivity in $\mathrm{CH}$ selection. The main objective of our proposal is to allow nodes with a high degree of connectivity to become $\mathrm{CHs}$ and hence elect a lesser number of, and more efficient, CHs. CDEEC exploits the aggregation operation at $\mathrm{CH}$ level and incorporates a routing protocol inside clusters 
to conserve further energy, thereby extending the network lifetime.

The remainder of this paper is organized as follows. Section II presents the related work. In Section III, we expose the network model. Section IV describes the proposed clustering protocol, followed by the performance evaluation in section $\mathrm{V}$. Finally, section VI contains our concluding remarks.

\section{RELATED WORK}

Recently there has been increased interest in studying energy-efficient clustering algorithms in the context of both ad hoc and sensor networks. The main aim of clustering protocols in ad hoc networks is to generate the minimum number of clusters while maintaining network connectivity. In these algorithms the election of $\mathrm{CHs}$ is based mainly on the identity of nodes [15], the degree of connectivity [16] or the connected dominating set [17]. These techniques are discussed in depth in [6].

In the case of WSNs, the main objective of clustering protocols is to minimize energy consumption by the network in order to extend the network lifetime (surveys dealing with WSN clustering protocols can be found in [3] and [4]).

We classify WSN clustering protocols into two categories: probabilistic, in which a node becomes a $\mathrm{CH}$ with a certain probability, and deterministic, which requires an exchange of overhead messages for the CH's election. Here, we review some of the protocols proposed in the literature for each class: PEGASIS [18], DWEHC [21], and TASC [22] in the deterministic class, and EEHC [20], EECS [19], and HEED [8] in the probabilistic class.

Power-Efficient GAthering in Sensor Information Systems (PEGASIS) [18] is composed of two phases: 1) steady and 2) gathering. The steady phase consists of a formation of chains instead of clusters. In the chain formation, the BS and sensor nodes are connected via a chain using a greedy algorithm. One of the nodes, in the chain, is selected in turns to represent the head. In the data-gathering phase, each node delivers the sensing data to the nearest neighboring node until the data reaches the head node which aggregates and delivers the sensing data to the BS.

Distributed Weight-Based Energy-Efficient Hierarchical Clustering (DWEHC) [21] is another deterministic clustering protocol, which aims at high energy efficiency by generating balanced cluster sizes and optimizing the intra-cluster topology. To become a $\mathrm{CH}$, in DWEHC, each sensor node calculates its weight after exchanging its geographic location with its neighbors. The weight is a function of the sensor's residual energy and the distance to its neighbors. In a neighborhood, the node with the highest weight would be elected as a $\mathrm{CH}$ and the remaining nodes become CMs. The CMs at this stage are considered as first level because they are attached directly to the $\mathrm{CH}$. Then, the $\mathrm{CMs}$ progressively adjust their level to reach a $\mathrm{CH}$ using the minimum amount of energy. Specifically, if a $\mathrm{CM}$ finds an intermediary node with the minimum energy cost to reach its $\mathrm{CH}$, then it can choose this intermediary node as its parent.
Similar to the idea in [21], Topology Adaptive Spatial Clustering (TASC) [22] decomposes large non-uniform networks into smaller locally-uniform clusters. TASC assumes nodes are aware of 2-hop neighborhood information and also that they know the distance to their neighborhood. This is achieved by deriving a set of weights that includes distance, connectivity and density information within the locality of each node. The derived weights form the terrain for holding a $\mathrm{CH}$ election procedure in which each node selects the node closer to the center of mass of its 2-hop neighborhood to becoming its $\mathrm{CH}$.

Energy-Efficient Hierarchical Clustering (EEHC) [20] is a probabilistic clustering algorithm. The basic operation of the EEHC algorithm consists of electing $\mathrm{CHs}$ with probability $p$ and each $\mathrm{CH}$ announces its election to the k-hop away neighboring nodes. Any node that receives such a $\mathrm{CH}$ election announcement, if it is not itself a $\mathrm{CH}$, becomes a member of the closest cluster. In addition, if the election announcement does not reach a node within a specific time interval, the node becomes a forced $\mathrm{CH}$. However EEHC is extended to a corresponding multi-level architecture. In fact, the basic clustering process is recursively triggered at different steps. At each step $i$, EEHC elects levels of $\mathrm{CHs}$ with a corresponding election probability $p_{i}$, and a forwarding parameter $k_{i}$. The communication flow in EEHC is as follows. Ordinary sensor nodes, nodes that are not elected as $\mathrm{CHs}$, transmit their collected data to the corresponding first-level (level 1) $\mathrm{CHs}$, the $\mathrm{CHs}$ of the first-level clusters transmit the aggregated data to the second-level $\mathrm{CHs}$ and so on, till the highest level, $h$, of the clustering hierarchy is reached. Then the $\mathrm{CHs}$ of those $h$ level clusters transmit their final aggregated data reports to the BS.

In [19], the authors proposed the Energy-Efficient Clustering Scheme (EECS) protocol. In this protocol, $\mathrm{CH}$ candidates compete for the ability to elevate to a $\mathrm{CH}$ with a certain probability. This competition involves candidates broadcasting their residual energy to neighboring candidates. If a given node does not find a node with more residual energy, it becomes a $\mathrm{CH}$. EECS extends this algorithm by the dynamic sizing of clusters based on cluster distance from the BS. The result is an algorithm that addresses the problem that clusters at a greater range from the BS require more energy for transmission than those that are closer. Ultimately, this improves energy distribution throughout the network, resulting in better resource usage and extended network lifetime.

Hybrid, Energy-Efficient Distributed Clustering (HEED) [8] is one of the well-known clustering protocols in WSNs, and on which CDEEC is built. The HEED protocol operates in two main phases: (1) the set-up phase where clusters are formed and (2) the steady phase where the sensor nodes transmit their data using Time Division Multiple Access (TDMA) frames. The choice of the CHs with HEED is made in an iterative way. The aim is to achieve a better $\mathrm{CH}$ distribution within the WSN, but this is made at the cost of more complexity and increased $\mathrm{CH}$ overhead within the WSN.

The first sub-phase is the initialization. Nodes exchange hello messages to discover their neighborhoods. The second 
sub-phase consists of a competition process and allows the election of $\mathrm{CHs}$ in the network. The third sub-phase is the finalization and allows nodes to join their corresponding $\mathrm{CH}$ based on the degree of connectivity.

Before a node starts executing HEED, it calculates its probability of becoming a $\mathrm{CH}, \mathrm{CH}_{\text {prob }}$, as follows:

$$
C H_{\text {prob }}=C_{\text {prob }} \times \frac{E_{\text {residual }}}{E_{\text {max }}}
$$

where $C_{\text {prob }}$ is an initial percentage of $\mathrm{CHs}$ among all nodes, $E_{\text {residual }}$ is the current node residual energy and $E_{\max }$ is a reference maximum energy.

The clustering process requires a number of iterations at each node. During iteration $i$, every uncovered node that does not hear any $\mathrm{CH}$ notification from its neighbors, elects itself as a $\mathrm{CH}$ with a probability $\mathrm{CH}_{\text {prob }}$. After iteration $i$, the set of potential CHs, $S_{C H}$, is set to: $\{\mathrm{CHs}$ after iteration $i-1 \bigcup$ new $\mathrm{CH}$ in iteration $i\}$. A node $n_{j}$ joins the $\mathrm{CHs}$ with the lowest cost in $S_{C H} . S_{C H}$ may include $n_{j}$ itself if it is selected as a tentative $\mathrm{CH}$. Then, every node doubles its $\mathrm{CH}_{\text {prob }}$ and goes to the next iteration.

If a node elects itself as a $\mathrm{CH}$, it sends a tentative message including node ID, status and cost. If a node completes HEED execution without being assigned to a final $\mathrm{CH}$, it considers itself uncovered and announces itself as a $\mathrm{CH}$ with final state.

However, the election of CHs in HEED does not take into a consideration the degree of connectivity, which can increase the efficiency of the $\mathrm{CH}$ election. We propose a mechanism that allows nodes with a high degree of connectivity to become $\mathrm{CHs}$ and, hence benefit from the aggregation operation at $\mathrm{CH}$ level to conserve more energy. In addition, as HEED does not implement a routing scheme inside the clusters, we extend the HEED's intra-cluster communication by incorporating a routing protocol within a cluster.

\section{NETWORK MODEL}

We consider a WSN consisting of $N$ sensors deployed over a vast field to continually monitor the environment. We denote the $i$-th sensor node by $n_{i}$. We use the following assumptions concerning sensor nodes and the underlying network model:

- Sensor nodes and the BS are stationary after deployment. The BS is far away from the square sensing field and can be reached by sensor nodes under a single high transmission range $T R 2$.

- Nodes are dispersed in the $A \times A$ area. Nodes are homogenous and have the same capabilities. A unique identifier ID is assigned to each node.

- Each node $n_{i}$ can reach its neighbors $n_{j}\left(n_{j} \in\right.$ Set_neighbor ${ }_{i}$, where Set_neighbor ${ }_{i}$ denotes the set of $n_{i}$ ' neighbors) and the BS with transmission ranges $T R 1$ and $T R 2$, respectively $(T R 1<T R 2)$. We refer here to $T R 1$ and $T R 2$ as the minimum and the maximum energy level available at the sensor node, respectively, in which nodes can choose to reach the required destination.

- Links are symmetric, i.e., if $n_{i} \in$ Set_neighbor ${ }_{j}$, then $n_{j} \in$ Set_neighbor ${ }_{i}$.
- Aggregation is performed only at $\mathrm{CH}$ level. $\mathrm{CHs}$ use perfect aggregation to eliminate data redundancy and reduce communication load i.e., any number of packets can be aggregated to one packet [9]. Other aggregation techniques, such as those proposed in our previous work [10], are still possible.

In order to estimate the mote's residual energy, we use the energy model from Heinzelman et al [9]. We assume that only transmission, reception and aggregation consume energy. No energy is consumed during sleep mode. According to this model, a sensor spends $E_{\text {elec }}=50 \mathrm{~nJ}$ to run the transmitter receiver circuitry. To transmit a packet of size $k$ over a distance $d$, the amount of energy consumed $E_{T x}$ is given by the following equation.

$$
E_{T x}=\left\{\begin{array}{l}
\left(E_{\text {elec }} * k\right)+E_{f s} * k * d^{2}, \text { if } d<d_{0} \\
\left(E_{\text {elec }} * k\right)+E_{m p} * * d^{4}, \text { otherwise. }
\end{array}\right.
$$

where $E_{m p}$ and $E_{f s}$ depends on the transmitter amplifier model to use, $d_{0}$ is the distance threshold between the transmitter and the receiver over which the multi-path fading channel model is used. To receive a message of size $k$, the energy required by the receiver is given by: $E_{R x}=\left(E_{\text {elec }} * k\right)$. The energy consumed in aggregating the data is $E_{D A}$.

\section{PROPOSED PROTOCOL}

This section describes our proposed protocol. Firstly, we study the case of a simple topology using HEED, and then we provide some hints on our proposed CDEEC protocol used to alleviate issues that may arise within HEED. An additional mechanism is also provided in order to enhance the performance of our proposal in general topologies.

\section{A. A case study}

To illustrate the operation of HEED we use the simple topology presented in Fig. 1. Here, we iterate sub-phase two (i.e., CH selection sub-phase) of the HEED set-up phase. Tables I and II draw the different iterations for nodes $n_{1}$ and $n_{4}$, respectively. We note that nodes $n_{1}, n_{2}, n_{5}$ and $n_{6}$ behave similarly since they are symmetric. Likewise for nodes $n_{3}$ and $n_{4}$.

In our analysis we use the following notations. $\mathrm{CH}_{\text {previous }}$ denotes the probability of becoming a $\mathrm{CH}$ at the previous iteration i.e., iteration $i-1$. We set $C H_{\text {previous }}$ to 0.2 for all the nodes at iteration 0 .

In our example (see tables I and II), we assume that only nodes $n_{1}$ and $n_{5}$ access the medium and send tentative messages for their neighbors. Following each iteration the set $S_{C H}\left(n_{j}\right)$ of CHs, that are one hop away from node $n_{j}$

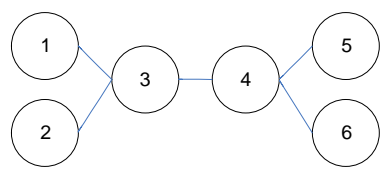

Fig. 1. Network topology. 
$(j=1, \ldots, N)$, is updated. After each iteration $i$, node $n_{j}$ doubles its $C H_{\text {prob }}$. Once $C H_{\text {previous }}$ of a node $n_{j}$ reaches 1 , it joins the least costly $\mathrm{CH}$ of the set $S_{C H}\left(n_{j}\right)$. Otherwise (i.e., $S_{C H}\left(n_{j}\right)=\emptyset$ ), node $n_{j}$ forces itself to become a $\mathrm{CH}$.

In our example, at the end of sub-phase two, nodes $n_{1}$ and $n_{5}$ become CHs. At the finalizing sub-phase (sub-phase three), nodes $n_{3}$ and $n_{4}$ join their $\mathrm{CHs}$ nodes $n_{1}$ and $n_{5}$, respectively. On the other hand, nodes $n_{2}$ and $n_{6}$ force themselves to become CHs since $S_{C H}(2)=\emptyset$ and $S_{C H}(6)=\emptyset$.

It is worth noting that with HEED the cost of a $\mathrm{CH}$ is considered to be its degree of connectivity (i.e., the count of neighbors it has). Hence, nodes with a lower degree of connectivity are preferred to become CHs.

It is also important to highlight that with HEED, once a node $n_{j}$ sends a tentative message at iteration $i$ to possibly become a $\mathrm{CH}$, it prevents its neighbors henceforth from sending tentative messages (i.e., from competing to become $\mathrm{CHs}$ ). As a result the first node among its neighborhood that succeeds in sending a tentative message becomes the $\mathrm{CH}$ for its neighbors. If many nodes send tentative messages at the same iteration, the least costly nodes become $\mathrm{CHs}$.

Intuitively, with HEED, nodes with a high degrees of connectivity have a low probability of becoming CHs. This is due to two main reasons:

- Firstly, there is a high probability that one of its neighbors sends a tentative message before the highly-connected node. This probability increases, the higher the nodes' degree of connectivity.

- Secondly, if a highly-connected node succeeds in sending a tentative message at the same iteration as some of its neighbors, the least-costly node (i.e., the node with the lowest degree of connectivity) will be elected as a $\mathrm{CH}$.

To illustrate this again, let us consider the following star network example presented in Fig. 2. Suppose that all the $N$ nodes have the same $\mathrm{CH}_{\text {prob }}$ at the beginning denoted by $p$. It is easy to see that once one of the leaf nodes sends a tentative message, it prevents the node at the center $n_{0}$ from becoming a $\mathrm{CH}$. Specifically, the probability that node $n_{0}$ is elected as a $\mathrm{CH}$ among its neighbors can be written as follows:

$$
\operatorname{Prob}\{A \mid B\}=\frac{p(1-p)^{N-1}}{1-(1-p)^{N}}
$$

where the events $A$ and $B$ are:

$A=\left\{n_{0}=\mathrm{a} \mathrm{CH}\right\}$.

$B=\{$ there is at least one node that has sent a tentative message $\}$, and thus the probability that leaf nodes become CHs is:

TABLE I

ITERATION OF NODE 1 .

\begin{tabular}{|c|c|c|l|}
\hline iteration & $\mathrm{CH}_{\text {previous }}$ & $\mathrm{CH}_{\text {prob }}$ & $S_{C H}$ \\
\hline 0 & 0.2 & 0.2 & $\emptyset$ \\
\hline 1 & 0.2 & 0.4 & 1 \\
\hline 2 & 0.4 & 0.8 & 1 \\
\hline 3 & 0.8 & 1 & 1 , final message \\
\hline 4 & 1 & 1 & 1, final message \\
\hline
\end{tabular}

TABLE II

ITERATION OF NODE 4

\begin{tabular}{|c|c|c|l|}
\hline iteration & $\mathrm{CH}_{\text {previous }}$ & $\mathrm{CH}_{\text {prob }}$ & $S_{C H}$ \\
\hline 0 & 0.2 & 0.2 & $\emptyset$ \\
\hline 1 & 0.2 & 0.4 & 5 \\
\hline 2 & 0.4 & 0.8 & 5 \\
\hline 3 & 0.8 & 1 & 5, final message \\
\hline 4 & 1 & 1 & 5, final message \\
\hline
\end{tabular}

$$
\operatorname{Prob}\{\bar{A} \mid B\}=1-\frac{p(1-p)^{N-1}}{1-(1-p)^{N}}
$$

We can see that $\operatorname{Prob}\{A \mid B\} \ll \operatorname{Prob}\{\bar{A} \mid B\}$. In this case, all the leaf nodes become $\mathrm{CHs}$, and node $n_{0}$ will join one of them. Then, each of the leaf nodes has to send its report directly to the distant sink node, which results in excessive energy consumption. However if the node in the center, $n_{0}$, was elected as a $\mathrm{CH}$, it would aggregate all the data from the leaf node and send the aggregate packet at once to the sink node. As a result, high energy communication with the sink node is replaced by low energy and short distance communication with the $\mathrm{CH}$.

This example illustrates the limitations of the HEED, which may lead to excessive energy consumption. In what follows we propose our CDEEC protocol to cope with the aforementioned issue.

To alleviate the aforementioned problem and to allow the election of efficient $\mathrm{CHs}$ that maximally exploit the aggregation operation, we propose to block nodes with a lower degrees of connectivity (i.e., with a degree of connectivity $<M$, where $M$ is a pre-specified threshold) from competing to become CHs. This proposed technique is inspired by [13], in which nodes are blocked from $\mathrm{CH}$ competition with a predefined probability. By doing so, we aim to block nodes that are not suitable to become $\mathrm{CHs}$ in advance and to, therefore allow only targeted nodes to $\mathrm{CH}$ competition.

Obviously, a blocked node will force itself to become a $\mathrm{CH}$ at sub-phase three since its set of $\mathrm{CHs} S_{C H}=\emptyset$. To illustrate this let us revisit the example of Fig. 1. In this example we block nodes with a degree of connectivity < 2 from participating in the $\mathrm{CH}$ selection process i.e., we set $M=2$. In doing so nodes $n_{1}, n_{2}, n_{5}, n_{6}$ do not participate in the $\mathrm{CH}$ selection process. Only nodes $n_{3}$ and $n_{4}$ compete

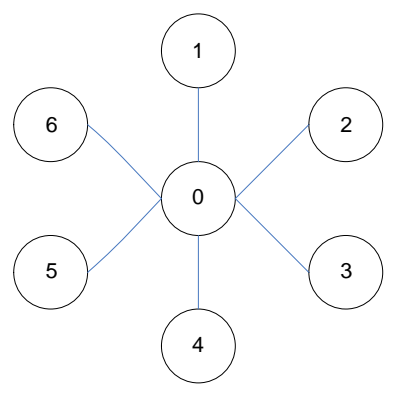

Fig. 2. Star topology. 
to become CHs. Suppose that node $n_{1}$ is elected as a $\mathrm{CH}$. In this case, nodes $n_{3}, n_{2}$ and $n_{4}$ will join $\mathrm{CH} n_{3}$ as Cluster Members (CMs). On the other hand, the sets of $\mathrm{CHs}$ associated with nodes $n_{5}$ and $n_{6}$ are null. As such, nodes $n_{5}$ and $n_{6}$ force themselves to become CHs. We can see that, with CDEEC, three nodes (either $\left\{n_{3}, n_{5}, n_{6}\right\}$ or $\left\{n_{1}, n_{2}, n_{4}\right\}$ ) are elected as CHs. On the other hand, using HEED, nodes $\left\{n_{1}, n_{2}, n_{5}\right.$, $\left.n_{6}\right\}$ (in addition to nodes $n_{3}$ and $n_{4}$ ) will compete from the beginning in the $\mathrm{CH}$ selection process. As such, the following four nodes $\left\{n_{1}, n_{2}, n_{5}, n_{6}\right\}$ are likely to become CHs. This results in increasing energy consumption in the network since it increases the number of high energy-consuming communications between the sensor nodes and the distant sink node (four $\mathrm{CHs}$ instead of three). Using our method, however, replaces one high energy consuming communication with a local and lower-consuming energy communication.

\section{B. Additional mechanism}

To further reduce the number of $\mathrm{CHs}$, we also propose to permanently block nodes with degrees of connectivity $<M$ from becoming CHs. To illustrate this, let us revisit Fig. 1. Recall that in this example only node $n_{3}$ is selected as a $\mathrm{CH}$ at the end of sub-phase two of the clustering process. Moreover nodes $n_{1}, n_{2}$ and $n_{4}$ join $\mathrm{CH} n_{3}$ as CMs. On the other hand, the sets of $\mathrm{CHs}$ associated with nodes $n_{5}$ and $n_{6}$ are null. Instead of forcing these nodes to become $\mathrm{CHs}$ at the end of the clustering process, we propose to let them join $\mathrm{CH} n_{3}$ by using a multi-hop routing. In other words, nodes $n_{5}$ and $n_{6}$ join $\mathrm{CH} n_{3}$ as CMs. Specifically, both nodes send their reports to the $\mathrm{CH} n_{3}$ through $n_{4}$.

In doing so, we replace the high energy-consuming communications between sensor nodes $n_{5}$ and $n_{6}$ with the distant sink node by local and short multi-hop communications with the $\mathrm{CH}$. As a result, the $\mathrm{CH} n_{3}$ is the only one responsible for communicating the aggregated data to the distant sink node. Compared to HEED, we can see that energy conservation can be realized. Based on this simple example, only one $\mathrm{CH}$ is elected instead of four by enabling our scheme. In other words, we increase the aggregation capacity of the elected $\mathrm{CH}$ as, instead of four packets, only one is sent to the sink node.

Generally, in order to avoid isolating blocked nodes from the networks a blocked node, $n_{i}$, can decide to unblock itself, following two conditions:

1) If all of $n_{i}$ 's neighbors are not participating in the clustering (due to dead nodes or is the blocking process), and

2) If $n_{i}$ has the highest degree of connectivity among its neighbors (in case of the same degree of connectivity, they all decide to participate in the clustering process).

By doing so, we ensure at least one unblocked neighbor for any $n_{i}$. As a result, the blocked node chooses one of its neighbors as a next-hop node as follows:

- If $n_{i}$ hears final messages from $\mathrm{CHs}$, it registers itself as a $\mathrm{CM}$ and joins the node with the highest cost among the $\mathrm{CH}$ announcements.
- If $n_{i}$ does not hear any final message, it chooses one of the unblocked neighbors as the next-hop.

\section{Performance eValuation}

In this section, we describe the results of simulations carried out. To achieve this, we use TOSSIM under TinyOS [14]. The application we use in our study is a continuous monitoring application, where data is generated continuously with a predefined reporting frequency. Nodes can use two different levels of power depending on the type of transmissions. On one hand, CMs use transmission range $T R 1$ to reach $\mathrm{CHs}$. On the other hand, $\mathrm{CHs}$ use transmission range $T R 2$ to reach the $\mathrm{BS}$. The clustering process is triggered every round time in order to balance the energy consumption of $\mathrm{CHs}$. Therefore, for every round time, new $\mathrm{CHs}$ and $\mathrm{CMs}$ are elected. The network is configured with the parameters listed in Table III.

TABLE III

Simulation PARAMETERS.

\begin{tabular}{|l|l|}
\hline Parameter & value \\
\hline$E_{f s}$ & $10 \mathrm{pJ} /$ bit $/ \mathrm{m}^{2}$ \\
\hline$E_{m p}$ & $0.0013 \mathrm{~nJ} / \mathrm{bit} / \mathrm{m}^{4}$ \\
\hline$E_{\text {elec }}$ & $50 \mathrm{~nJ} / \mathrm{bit}$ \\
\hline$E_{D A}$ & $5 \mathrm{~nJ} / \mathrm{bit} /$ signal \\
\hline Threshold $d_{0}$ & $25 \mathrm{~m}$ \\
\hline Initial energy per node & $0.05 \mathrm{~J}$ \\
\hline Transmission bit rate & $40 \mathrm{kbs}^{-1}$ \\
\hline Time slot & $0.014976 \mathrm{sec}$ \\
\hline Round time & $1000 \mathrm{sec}$ \\
\hline Monitoring frequency & $100 \mathrm{sec}$ \\
\hline TR1 & $20 \mathrm{~m}$ \\
\hline$T R 2$ & $300 \mathrm{~m}$ \\
\hline Area & $100 * 100 \mathrm{~m}^{2}$ \\
\hline
\end{tabular}

\section{A. Simulation results}

Firstly, we show the results concerning the case study that has already been described in subsection IV-A. Then, we show the results concerning the additional mechanism generalized into random topologies.

1) Case study: Fig. 3(a) and Fig. 3(b) show the probability of becoming a $\mathrm{CH}$ for each node for both HEED and CDEEC protocols. As explained before, the probability of nodes $n_{3}$ and $n_{4}$ in CDEEC is higher than that of nodes in HEED. This is because of blocking nodes $n_{1}, n_{2}, n_{5}$ and $n_{6}$ from participating in the cluster process. The probability of node $n_{3}$ is higher than that of node $n_{4}$ since the priority of becoming $\mathrm{CHs}$ is given to nodes with a smaller node ID, in case of those with the same degree of connectivity.

Fig. 4 shows the average network residual energy over time. The result clearly demonstrates that CDEEC protocol conserves more energy, when compared to HEED.

The small number of reports received at the BS (see Fig. 5) in CDEEC compared to HEED can be explained by the fact that only a small number of $\mathrm{CHs}$ are elected, thereby reducing the amount of data to be transmitted and thus saving energy. This does not mean that less information is reported to the BS. Rather it means that CDEEC benefits from aggregation operation. The small difference in the number of reports issued 


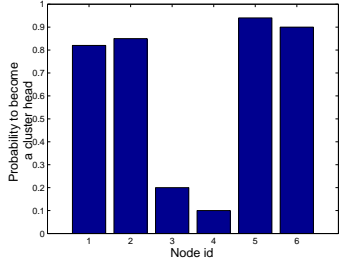

(a) HEED protocol.

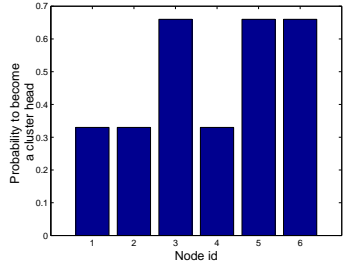

(b) CDEEC protocol.
Fig. 3. Probability of becoming a CH vs node ID.

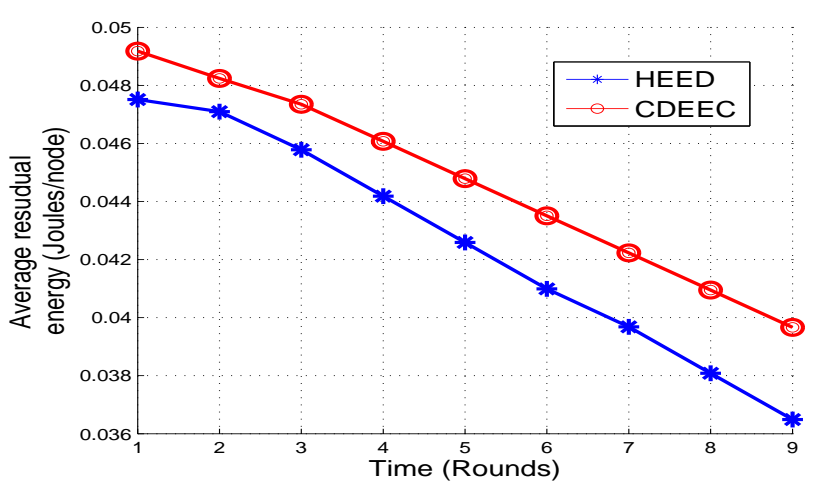

Fig. 4. Average residual energy of the network.

using the two protocols is explained by the fact that a small number of nodes are used in the network, and thus a small number of $\mathrm{CHs}$ are elected.

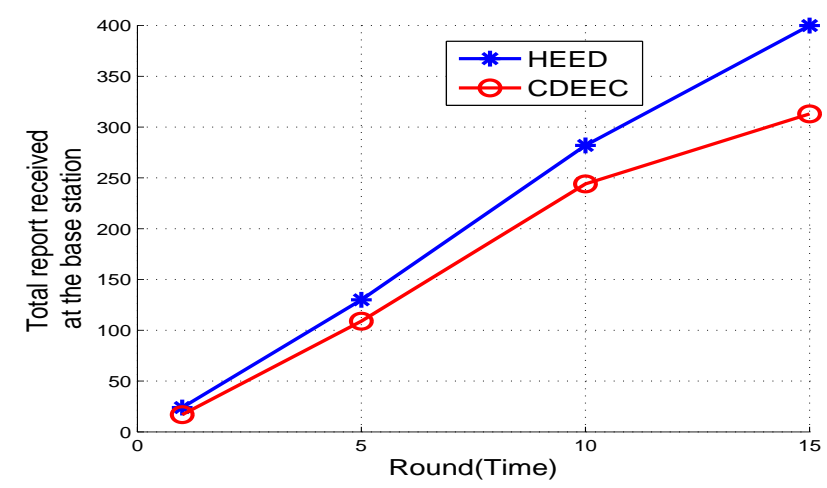

Fig. 5. Total reports received at the BS Vs time.

Finally, Fig. 6 depicts the average energy consumed for both protocols. As we can see, CDEEC saves further energy at the expense of high energy consumption of nodes with a high degree of connectivity. Recall that energy balancing is achieved by reclustering the network in each round. Therefore, nodes that are elected at any given round will have little chance of becoming $\mathrm{CHs}$ in the next round as $\mathrm{CH}$ election depends highly on the residual energy of nodes.

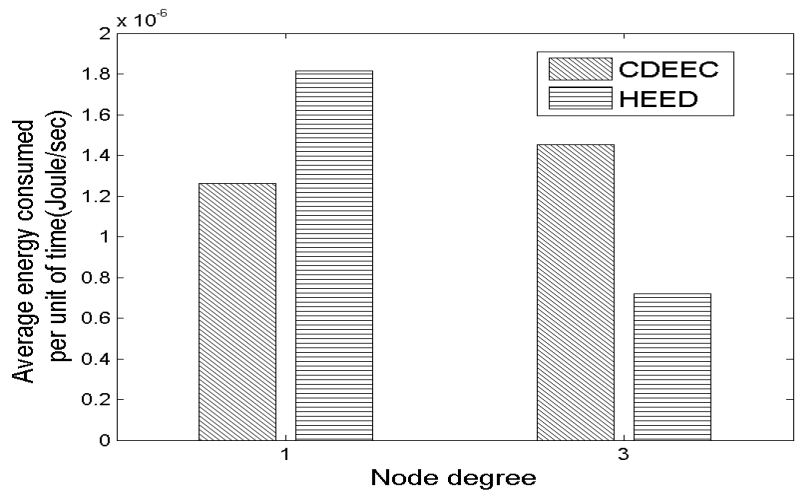

Fig. 6. Average energy consumed Vs Node degree.

2) Additional mechanism: The results in this section concern our CDEEC protocol while considering the generalized additional mechanism using random topologies.

To validate the performance of CDEEC we used random topologies rather than specific topologies. Three random topologies of 20 nodes were considered. These topologies differ based on the ACD parameter, defined as the Average Connectivity Degree of the network. As the proposed protocol is tightly based on the degree of connectivity, considering random topologies with different ACD parameter is sufficient to validate the results. Therefore we use different values of TR1: $20 \mathrm{~m}, 35 \mathrm{~m}$ and $55 \mathrm{~m}$ to create three random topologies of different connectivities: small (i.e., $\mathrm{ACD}=2$ ), moderate (i.e., $\mathrm{ACD}=6.2$ ) and highly-connected networks (i.e., $\mathrm{ACD}=10$ ), respectively.

We set the pre-specified threshold M to ACD. Fig. 7 and Fig. 8 show the effect of $M$ on both protocols. We can see that HEED is suitable for high-connected topologies. The reason is that more $\mathrm{CHs}$ are elected with small ACD value in HEED and therefore consume excessive energy. In contrast, CDEEC outperforms HEED since it restricts the percentage of $\mathrm{CHs}$. We can also see from these figures that CDEEC performs better in the topology with $A C D=6.2$ than in the topology with $A C D=10$. Though in these situations almost the same percentage of $\mathrm{CHs}$ are formed, the better performance of the $A C D=6.2$ case is achieved due to the fact that nodes use a smaller transmission range compared to the other topology. The choice of the threshold $M$ limits the number of nodes that will participate in the clustering process, which has a direct effect on the multi-hop routing process. Indeed, with a small $M$ value, HEED is preferred as the majority of nodes will participate in the clustering process and tend not to use a multihop routing inside the clusters. However, choosing a high value for $M$ decreases the number of nodes competing in clustering but at the same time makes the multi-hop process difficult to accomplish. This difficulty is related to whether the blocked nodes will be surrounded by at least one unblocked neighbor. Choosing an appropriate $M$ threshold requires knowledge of the network, such as the network structure (to specify which nodes are to be blocked or not), or the ACD parameter, as we 
have already seen.

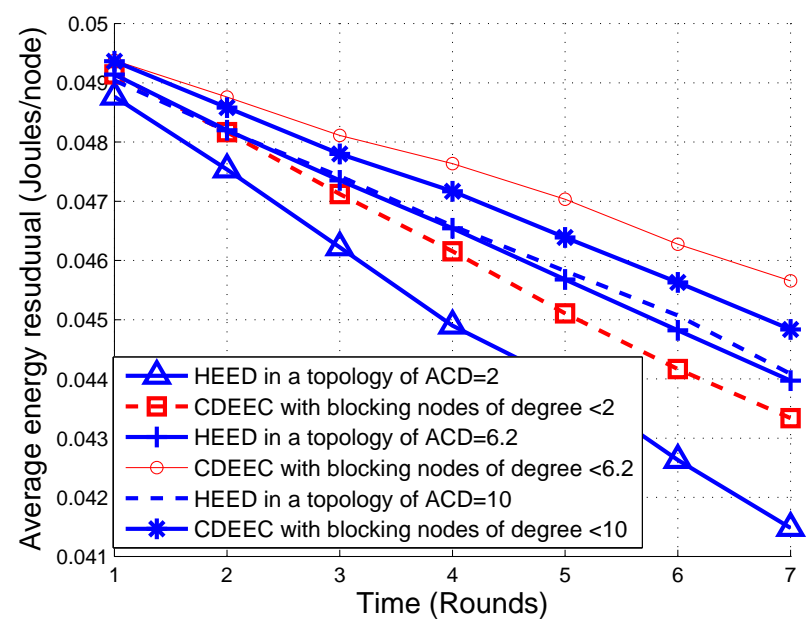

Fig. 7. Average energy consumed in the network.

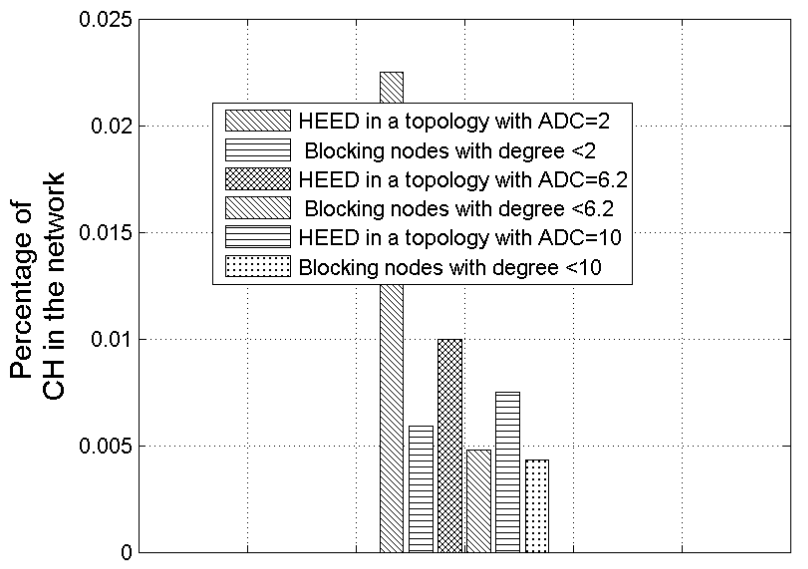

Fig. 8. Percentage of $\mathrm{CHs}$ in the network.

\section{CONCLUSION}

This paper aims at reducing energy consumption in wireless sensor networks. To achieve this, we considered the node's degree of connectivity, along with their residual energy, in the cluster head selection process. Specifically, we increased the probability of becoming $\mathrm{CHs}$ for nodes with a high degree of connectivity. By doing so, we benefit from the aggregation capacity of such nodes, thus enabling additional energy conservation. To evaluate the performance of our proposed CDEEC protocol, we implemented CDEEC in TinyOS and compared it with HEED. Results showed that significant energy conservation can be achieved.

\section{REFERENCES}

[1] J. N. Al-Karaki, and A. E. Kamal, "Routing techniques in wireless sensor networks: a survey," IEEE Wireless Communications, vol. 11, no. 6, pp. 6-28, December 2004.
[2] D. Estrin, D. Culler, K. Pister, and G. Sukhatme, "Connecting the physical world with pervasive networks," IEEE pervasive computing, pp. 59-69, January-Marche 2002.

[3] O. Younis, M. Krunz, and S. Ramasubramanian,"Node clustering in wireless sensor networks: Recent developments and deployment challenges IEEE Network," vol. 20, no. 3, pp. 2025, May-June. 2006.

[4] A. Abbasi, M. Younis, "A survey on clustering algorithms for wireless sensor networks, Journal of Computer Communications," Elsevier Computer Communications, vol. 30, pp. 2826-2841, 2007.

[5] V. Mhatre and C. Rosenberg, "Design guidelines for wireless sensor networks: communication, clustering and aggregation," Elsevier Ad Hoc Networks Journal, vol. 2, pp. 45-63, 2004.

[6] J.Y. Yu, and P.H.J. Chong, "A survey of Clustering schemes for Mobile Ad hoc Networks," IEEE Communication Surveys and Tutorials, vol. 7, no. 1 , pp. 32-48, 2005.

[7] J. Neander, E. Hansen, M. Nolin, and M. Bjorkman, "Asymmetric multihop communication in large sensor networks," in Proc. International Symposium on Wireless Pervasive Computing 2006, Jan. 2006.

[8] O. Younis, and S. Fahmy, "HEED: A hybrid, energy-efficient, distributed clustering approach for ad-hoc sensor networks," IEEE Transactions on Mobile Computing, vol. 3, no. 4, pp. 366-379, October-December 2004.

[9] W. Heinzelman, A. Chandrakasan, and H. Balakrishanan, "An Application-Specific Protocol Architecture for Wireless Microsensor Networks," IEEE Transactions on Wireless Communications, vol. 1, no. 4, pp. 660-670, 2002.

[10] M. Abdul Azim, S. Moad and N. Bouabdallah,"SAG: Smart Aggregation Technique for Continuous-Monitoring in Wireless Sensor Networks", International Conference on Communications (ICC), Captown, May 2010.

[11] D. M. Blough and P. Santi, "Investigating Upper Bounds on Network Lifetime Extension for Cell-Based Energy Conservation Techniques in Stationary Ad Hoc Networks," in Proc. ACM/IEEE International Conference on Mobile Computing and Networking (MOBICOM), 2002.

[12] I. Dietrich and F. Dressler, "On the Lifetime of Wireless Sensor Networks", ACM Transactions on Sensor Networks, vol. 5, no. 1, February 2009.

[13] M. Ye, C. Li, G. Chen, and J. Wu, "EECS: An energy Efficient Clustering Scheme in wireless Seneor networks," National Laboratory of Novel Software Technology, Nanajing University, China, 2005.

[14] P. Levis, N. Lee, M. Welsh, and D. Culler, "TOSSIM: Accurate and Scalable Simulation of Entire TinyOS Applications," in Proc. ACM Conference on Embedded Networked Sensor Systems (SenSys), 2003.

[15] D. J. Baker, and A. Ephremides, "The architectural organization of a mobile radio network via a distributed algorithm," IEEE Transactions on Communications, vol. 29, no.11, pp. 1694-1701, 1981.

[16] M. Gerla, and J.T.C. Tsai, "Multicluster, mobile, multimedia radio network," ACM/Baltzer Journal of Wireless Networks, vol. 1, no. 3 , pp. 255-265, 1995.

rete Algorithms and Methods for Mobile Computing and Communications, pp. 7-14, 1999.

[17] P. J. Wan, K. M. Alzoub, and O. Frieder, "Distributed construction of connected dominating set in wireless ad hoc networks," in Proc. IEEE INFOCOM Conference, New York, June 23-27, 2002.

[18] Lindsey, S. and Raghavendra, C.S.,"PEGASIS: Power-efficient gathering in sensor information systems," IEEE Aerospace Conference Proceedings, vol. 3, pp. 3-1125, 2002

[19] Ye, M. and Li, C. and Chen, G. and Wu, J., "EECS: an energy efficient clustering scheme in wireless sensor networks," 24th IEEE International Performance, Computing, and Communications Conference, pp. 535540, 2007

[20] Bandyopadhyay, S. and Coyle, E.J., "An energy efficient hierarchical clustering algorithm for wireless sensor networks," INFOCOM 2003. Twenty-Second Annual Joint Conference of the IEEE Computer and Communications. IEEE Societies, vol. 3 pp 1713-1723, 2003.

[21] Ding, P. and Holliday, J.A. and Celik, A., "Distributed energy-efficient hierarchical clustering for wireless sensor networks," Distributed Computing in Sensor Systems springer jounral, pp. 322-339, 2005

[22] Virrankoski, R. and Savvidees, A., "TASC: topology adaptive spatial clustering for sensor networks," IEEE International Conference on Mobile Adhoc and Sensor Systems Conference, pp. 614, 2005 